\title{
artigo
}

Amorim, G.; Ferreira, D.C.; Miranda, V.O.; Santos, D.C.; Vieira, M.P.;

Conhecimento de indivíduos diabéticos sobre os benefícios e fontes da fibra alimentar

\section{Conhecimento de indivíduos diabéticos sobre os benefícios e fontes da fibra alimentar}

Knowledge of diabetic individuals about the benefits and sources of food fiber

Conocimiento de personas diabéticas sobre los beneficios y fuentes de la fibra alimentaria

\section{RESUMO}

Este estudo avaliou o nível de conhecimento sobre Fibras Alimentares em indivíduos diabéticos. Trata-se de um estudo epidemiológico transversal. Os dados foram coletados através de questionários. Alguns participantes apresentaram conhecimentos superficiais sobre as fibras alimentares. O nutricionista é essencial nas Unidades Básicas de Saúde para fortalecer o acesso à informação. DESCRITORES: Diabetes Mellitus; Estado Nutricional; Dieta Saudável; Fibras na Dieta.

\section{ABSTRACT}

This study assessed the level of knowledge about dietary fiber in diabetic individuals. This is a cross-sectional epidemiological study. The data were collected through questionnaires. Some participants presented superficial knowledge about dietary fibers. The nutritionist is essential in the Basic Health Units to strengthen access to information.

DESCRIPTORS: Diabetes Mellitus; Nutritional Status; Diet Healthy, Dietary Fiber.

\section{RESUMEN}

Este estudio evaluó el nivel de conocimiento sobre la fibra dietética en personas diabéticas. Este es un estudio epidemiológico transversal. Los datos fueron recolectados a través de cuestionarios. Algunos participantes presentaron conocimientos superficiales sobre las fibras dietéticas. El nutricionista es fundamental en las Unidades Básicas de Salud para fortalecer el acceso a la información.

DESCRIPTORES: Diabetes Mellitus; Estado Nutricional; Dieta Saludable; Fibras de la Dieta.

RECEBIDO EM: 15/10/2020 APROVADO EM: 26/10/2020

\section{Gláucia Amorim}

Graduada em Nutrição pela Universidade Federal de Juiz de Fora - Campus Governador Valadares. Nutricionista do Núcleo de Apoio à Saúde da Família (NASF) no Distrito de Conceição de Tronqueiras - Coroaci - MG.

ORCID: 0000-0001-8370-2953

\section{Daniela Corrêa Ferreira}

Graduada em Nutrição pela Universidade Federal de Ouro Preto, especialista em Nutrição Clínica pela Universidade Veiga de Almeida. Mestre e Doutora em Ciência de Alimentos pela Universidade Federal de Minais Gerais. Professora Adjunta do Departamento do Curso de Nutrição da Universidade Federal de Juiz de Fora - Campus Governador Valadares.

ORCID: 0000-0002-1480-2974

\section{Vanessa de Oliveira Miranda}

Graduada em Enfermagem pela Universidade Vale do Rio Doce - Enfermeira da Estratégia Saúde da Família Sebastião Andrade (ESF-SA) no Distrito de Conceição de Tronqueiras - Coroaci - MG.

ORCID: 0000-0002-7420-4263

\section{Deysimara Cássia Santos}

Discente do curso de Nutrição na Universidade Federal de Juiz de Fora, bolsista do Programa de Educação pelo Trabalho para a Saúde Pet/Saúde - Interprofissionalidade UFJF- GV/SMS-GV, monitora da disciplina Gestão de Unidade de Alimentação I e ligante da Liga Acadêmica Multiprofissional de Promoção de Saúde Infantil.

ORCID: 0000-0001-7472-9222 


\section{Maisa Pereira Vieira}

Acadêmica de Nutrição na Universidade Federal de Juiz de Fora, componente do grupo de pesquisa Núcleo de Estudos da Pessoa Idosa. É extensionista no projeto Ação, Cidadania, Juventude e Comunidade - Turmalina (Programa Boa Vizinhança - Rio Doce) é também ligante da Liga Acadêmica Multiprofissional de Promoção de Saúde Infantil, bolsista no projeto de Extensão Estratégias de intervenção para o envelhecimento ativo na comunidade - Grupo operativo de apoio ao emagrecimento sustentável e alimentação.

ORCID: 0000-0003-1127-9353

\section{INTRODUÇÃO}

0 Diabetes Mellitus (DM), têm apresentado alta prevalência e incidência na população, estima-se que em 2040, cerca de 642 milhões de pessoas no mundo terão a doença ${ }^{1}$. Este consiste em um distúrbio metabólico caracterizado por hiperglicemia persistente, decorrente da deficiência na produção de insulina ou na sua ação, ou em ambos os mecanismos. O DM é classificado em Diabetes Mellitus tipo 1 (DM1) de etiologia autoinmune, Diabetes Mellitus tipo 2 (DM2) e Diabetes Mellitus gestacional ${ }^{2}$. Devido a seu caráter crônico, o DM é uma condição onerosa tanto para os pacientes quanto para o sistema de saúde . $^{3}$.

Sabe-se que a mudança no estilo de vida é essencial para prevenir as complicações do DM, além de auxiliar na promoção de um adequado controle glicêmi$\mathrm{co}^{4}$. Assim, a adoção de uma alimentação saudável, especificamente, atendendo as recomendações de fibras alimentares (FA) pode representar benefícios para os portadores de DM. A influência positiva das FA nos fatores determinantes da saúde. ${ }^{5.6}$, como a manutenção da microbiota intestinal ${ }^{7,8}$ está cada vez mais consolidada, entretanto, o Brasil ainda mantém um consumo inadequado desse nutriente ${ }^{6}$.

As FA são um conjunto de substâncias de origem vegetal resistentes à ação de enzimas digestivas no organismo humano, podendo ser consideradas carboidratos complexos, resistentes à digestão e absorção ${ }^{\text {. }}$. Estas podem ser classificadas em solúveis e insolúveis. As fibras solúveis (FS) dissolvem-se em água e não são digeridas no intestino delgado, estas têm efeitos benéficos em relação à glicemia. Já as fibras insolúveis (FI) possuem fermentação limitada, auxiliando no controle da saciedade e peso ${ }^{10.5}$.

O consumo adequado de alimentos fontes de FA trazem boas repercussões para a saúde do indivíduo, pois proporcionam uma dieta saudável e podem diminuir o risco de determinadas doenças $^{12}$. Assim, a Organização Mundial de Saúde (OMS) recomenda um consumo de $27-40 \mathrm{~g} /$ dia, já a SBD recomenda $14 \mathrm{~g} / 1.000 \mathrm{~g}$ diariamente ${ }^{13}$. O controle da glicemia é o foco principal no manejo da $\mathrm{DM}$, portanto, as técnicas que previnem a hiperglicemia tornam-se importante fator para a prevenção de possíveis complicações ${ }^{14}$.

Existem na literatura poucos estudos que exploram o grau de conhecimento e esclarecimento sobre o consumo de fibra alimentar pela população diabética. Nesse contexto, o presente trabalho teve como objetivo avaliar o nível de conhecimento de indivíduos diabéticos frequentadores de uma Unidade Básica de Saúde sobre a FA, em um distrito de Coroaci, Minas Gerais.

\section{MÉTOdO}

Trata-se de um estudo epidemiológico transversal de carácter exploratório, realizado com frequentadores da Estratégia de Saúde da Família (ESF) de Conceição de Tronqueiras, distrito de Coroaci - MG. Desta forma, buscou-se descrever o nível de conhecimento sobre os benefícios das fibras alimentares e seus principais alimentos fontes. $\mathrm{O}$ estudo foi realizado com 45 adultos e idosos diabéticos de ambos os sexos, cadastrados na ESF, no período de março a abril de 2018. A pesquisa foi aprovada pelo Comitê de Ética em Pesquisa em Seres Humanos da UFJF, sob o parecer 3.038838 e CAAE: 92489118.4.0000.5147. Após aceitarem participar, os indivíduos foram orientados a assinar o Termo de Consentimento Livre e Esclarecido. Os critérios de inclusão foram: indivíduos maiores de dezoito anos com diagnóstico de diabetes mellitus tipo 1, 2 e gestacional.

Para avaliação sociodemográfica, de saúde e a determinação dos conhecimentos acerca do consumo de fibras, utilizou-se questionário adaptado ${ }^{15.16 .17}$. Considerou-se porcentagem de acertos satisfatória um valor maior que $50 \%$. Além disso, foi fornecida uma intervenção educativa em forma de palestra. Aplicou-se um Questionário de Frequência Alimentar simplificado do tipo qualitativo, contendo alimentos separados por grupo alimentar, referente aos três últimos meses para registro. A frequência de consumo foi classificada em diária, mensal, duas ou três vezes na semana, quinzenal, raramente ou nunca ${ }^{17}$. Os alimentos contidos no questionário foram selecionados com base na Tabela Brasileira de Composição de Alimentos 2011, segundo a realidade de consumo da população. $\mathrm{O}$ consumo foi convertido em frequência diária equivalente (Diário $=1 ; 2$ a 3 vezes na semana $=0,35$; quinzenal $=0,06$; mensal $=0,03$; raro ou nunca $=0) 19$, obtendo-se o escore de ingestão diária para todos os alimentos.

$\mathrm{Na}$ avaliação antropométrica aferiu-se peso, altura, circunferência do pescoço (CP), circunferência da cintura (CC), Índice de massa corpórea (IMC) e relação cintura estatura (RCE). Para a medida da CP, os indivíduos foram posicionados de maneira ereta no plano horizontal e a medida foi realizada no ponto médio do pescoço, com a trena antropométrica, após isso classificou-se com elevado risco cardiovascular homens com CP $>35 \mathrm{~cm}$ e mulheres $\mathrm{CP}>34 \mathrm{~cm}$. 


\section{artigo}

Amorim, G.; Ferreira, D.C.; Miranda, V.O.; Santos, D.C.; Vieira, M.P.;

Conhecimento de indivíduos diabéticos sobre os benefícios e fontes da fibra alimentar

A CC foi aferida na região mais estreita do tronco ou, quando não aparente, no ponto médio entre a última costela e a borda superior da crista ilíaca ${ }^{20}$. Utilizou-se o ponto de corte proposto pela OMS (2004), onde valores iguais ou superiores a $94 \mathrm{~cm}$ e $80 \mathrm{~cm}$ representam risco aumentado de complicações metabólicas para homens e mulheres, respectivamente. O cálculo da RCE foi obtido através da razão do perímetro da cintura e a estatura, onde valor $>0,5$ foi classificado como alto risco ${ }^{21}$.

O IMC foi calculado utilizando peso e estatura aferidos, sendo os adultos classificados de acordo com a OMS, $(<18,4$ baixo peso, 18,5 a 24,9 estrófico, 25,0 a 29,9 sobrepeso e > 30,0 obesidade). Já para a classificação de idosos utilizou-se os valores preconizados por Lipschutz (1994), (22 kg/m² baixo peso, 22 a 27 $\mathrm{kg} / \mathrm{m}^{2}$ eutrófico e acima de $27 \mathrm{~kg} / \mathrm{m}^{2}$ sobrepeso).

O Teste Mann Whitney e Teste Kruskall Wallis foram utilizados para comparar mediana dos escores de frequência de consumo diário de alimentos fontes de fibras. O Teste de Qui-Quadrado de Pearson foi utilizado para verificar os fatores associados com o grau de conhecimento sobre fibras. O Teste de Mc Nemar foi utilizado para comparar as proporções de acertos das questóes antes e após a intervenção nutricional. Valores de $\mathrm{p}<0,05$ foram considerados estatisticamente significantes. Todas as análises foram concluídas usando o SPSS versão 20.0.

\section{RESULTADOS}

Realizou-se o estudo com 38 indivíduos diabéticos, sendo $14(36,8) \%$ adultos e $24(63,2 \%)$ idosos. A idade média do grupo estudado foi de 62,6 $\pm 15,4$ anos. $50 \%$ dos pacientes tinham ensino fundamental, $42,1 \%$ ensino médio, 5,3\% ensino superior e 2,6\% eram analfabetos. Em relação à atividade física 62,8\% eram sedentários. Como descrito pela Tabela 1- 65,85\% dos participantes são portadores de DM2, sendo que apenas $15,8 \%$ já fizeram acompanhamento nutricional.

Em relação ao IMC, 52,6\% dos par-

\section{Tabela 1 - Informações sobre o diabetes mellitus}

\section{Variáveis}

DM 1

DM 2

Diabetes gestacional

Tratamento

Dieta

Hipoglicemiante oral

Insulina

Hipoglicemiante oral + Insulina

Comorbidades

HAS

Não

Sim

Dislipidemia

Não

Sim

Acompanhamento nutricional

Sim

Não

\section{N \\ 25

\section{Tabela 2 - Estado nutricional e risco cardiometabólico.}

$\%$

31,6

65,8

2,6

10,5

65,8

7,9

15,8

16,2

83,8

81,1

18,9

15,8

84,2

IMC

Baixo peso

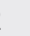

Eutrofia 


$\begin{array}{lcc}\text { Sobrepeso } & 12 & 31,6 \\ \text { Obeso grau I } & 3 & 7,9 \\ \text { Obeso grau II } & 1 & 2,6 \\ \text { Risco cardiometabólico segundo CC } & 11 & 28,9 \\ \text { Adequado } & 27 & 71,1 \\ \text { Risco elevado } & & 5,3 \\ \text { Risco cardiometabólico segundo RCE } & 2 & 94,7 \\ \text { Menor risco } & 36 & \\ \text { Alto risco } & & 18,4 \\ \text { Classificação do risco cardiometabólico segundo CP } & 7 & 81,6 \\ \text { Adequado } & 31 & \end{array}$

ticipantes encontravam-se eutróficos. Já

o risco cardiometabólico, $71,1 \%$ foram

classificados com risco elevado, 94,7\% alto risco e $81,6 \%$ risco elevado, de acordo com CC, RCE e CP, respectivamente.

Sobre nível de conhecimento FA ( Ta- bela 3), 57,9\% dos pacientes disseram que já ouviram falar em FA, além disso, $65,8 \%$ souberam citar alimentos fontes de FA.

\section{Tabela 3 - Percepção sobre alimentação e fibras.}

\section{Variáveis}

Já ouviram falar sobre a fibra

$\operatorname{Sim}$

Não

Souberam informar fonte de fibras

Sabe

Não sabe

Número de acertos

Não satisfatório (menor que 3)

Satisfatório ( $\geq 3$ )
N

22

16

25

13

17

21
$\%$

57,9

42,1

65,8

34,2

44,7

55,3

\section{Tabela 4 - Testes de associação}

\section{Variáveis}

Sexo

Masculino

Feminino

Idade

Adulto

Idoso

Escolaridade

Ensino fundamental

Ensino médio

Ensino superior
Total de acertos nas questões sobre FA (\%)

Não satisfatório Satisfatório

\section{0,508}

36,4

63,6

48,1

51,9

42,8

45,8

57,1

54,2

0,299

52,6

47,4

37,5

62,5

0,0
Os resultados da verificação da relação dos fatores associados com o conhecimento sobre a fibra alimentar estão descritos na Tabela 4 .

A Tabela 5 demonstra que, pacientes que recebem acompanhamento nutricional apresentam maior mediana no consumo de vegetais em comparação com os demais.

Nota: Valores de escore de frequência de consumo diário apresentados na forma de mediana e intervalo interquartil (p25 p75).* *Teste de Mann Whitney. ${ }^{* *}$ Teste de Kruskall Wall

Já a Tabela 6, compara o percentual de acertos às questões sobre saúde e sobre conhecimento de FA, entre os pacientes diabéticos antes e após a intervenção. 


\section{artigo}

Amorim, G.; Ferreira, D.C.; Miranda, V.O.; Santos, D.C.; Vieira, M.P.;

Conhecimento de indivíduos diabéticos sobre os benefícios e fontes da fibra alimentar

\begin{tabular}{|c|c|c|c|}
\hline Analfabeto & 100,0 & 0,0 & \\
\hline HAS & & & 0,715 \\
\hline Sim & 41,9 & 58,1 & \\
\hline Não & 50,0 & 50,0 & \\
\hline Dislipidemia & & & 0,410 \\
\hline Sim & 40 & 60,0 & \\
\hline Não & 57,1 & 42,9 & \\
\hline Tipo de Diabetes & & & 0,622 \\
\hline DM2 & 44,0 & 56,0 & \\
\hline DM1 & 50,0 & 50,0 & \\
\hline Diabetes Gestacional & 0,0 & 100,0 & \\
\hline Tratamento & & & 0,311 \\
\hline Dieta & 75,0 & 25,0 & \\
\hline Hipoglicemiante oral & 36,0 & 64,0 & \\
\hline Insulina & 33,3 & 66,7 & \\
\hline Hipoglicemiante oral + Insulina & 66,7 & 33,3 & \\
\hline Consideram ter uma alimentação saudável & & & 0,968 \\
\hline Sim & 45,5 & 54,5 & \\
\hline Não & 46,2 & 53,8 & \\
\hline Tempo de diagnóstico do diabetes & & & 0,492 \\
\hline$<3$ anos & 38,9 & 61,1 & \\
\hline$\geq 3$ anos & 50,0 & 50,0 & \\
\hline
\end{tabular}

\section{DISCUSSÃO}

Dos 38 diabéticos entrevistados, $71,1 \%$ eram do sexo feminino, característica semelhante a outros estudos, onde há maior predominância do sexo feminino10;16. A predominância de pacientes idosos no estudo pode ser justificada pelo aumento da prevalência da doença nesse grupo de indivíduos ${ }^{19}$.

A hipertensão está comumente associada ao diabetes e essa característica é evidente neste estudo, pois $68,8 \%$ dos participantes disseram ter a doença. Sabe-se que doenças cardiovasculares são a principal causa de morte nessa população, representando um importante problema de saúde pública. Portanto, a prevenção do DM, o gerenciamento da hiperglicemia e a prevenção de suas complicações é prioridade da saúde pública ${ }^{21}$.

Apesar dos pacientes apresentarem-se como eutróficos, verificou-se a presença de risco cardiometabólico por meio das variáveis de CC, RCE e CP. Já a CP aumentada está relacionada ao aumento

Tabela 5- Mediana e amplitude interquartil do escore de frequência de consumo diário para grupos alimentares

score de frequência de consumo diário

Variáveis

Sexo

Masculino

Feminino

Valor $\mathrm{p}^{*}$

Faixa etária

Adulto

Idoso

Valor $\mathrm{p}^{*}$

Escolaridade

Ensino médio

Ensino superior

Valor $p^{* *}$

Ocupação

Dona de casa/ aposentado

Trabalha

\section{Cereais Vegetais}

Frutas

Leguminosas

Alimentos fonte de fibras (escore total)

$$
\begin{array}{cc}
1,1(0,7-3,0) & 1,6(1,2-3,1) \\
1,1(0,7-2,1) & 2,1(1,3-2,8) \\
0,809 & 0,961
\end{array}
$$

$\begin{array}{cc}1,1(0,5-3,1) & 1,0(1,0-1,0) \\ 0,8(0,4-2,1) & 1,0(1,0-1,0) \\ 0,541 & 0,506\end{array}$

$6,0(3,2-10,0)$

$4,6(4,1-8,1)$

0,859

$$
\begin{gathered}
4,1(3,2-6,9) \\
6,2(4,2-8,5) \\
0,077
\end{gathered}
$$

$$
0,477
$$$$
0,058
$$

0,109

0,842

$$
\begin{array}{cc}
1,5(0,8-2,6) & 1,8(1,1-3,0) \\
1,9(1,1-2,7) & 2,9(2,8-3,1) \\
0,529 & 0,281
\end{array}
$$

$0,9(0,7-2,4)$

$1,0(1,0-1,0)$

$6,2(4,0-8,3)$

$2,0(1,0-3,1)$

$1,0(1,0-1,0)$

0,422

0,960

$8,0(6,0-10,0)$

$$
0,419
$$

$\begin{array}{llll}1,1(0,8-2,4) & 2,2(1,3-2,8) & 0,9(0,4-2,4) & 1,0(1,0-1,0) \\ 0,9(0,6-3,1) & 1,5(1,0-2,9) & 0,9(0,7-2,6) & 1,0(1,0-1,0)\end{array}$

$4,7(4,1-8,3)$ $5,0(3,2-8,6)$ 


\begin{tabular}{|c|c|c|c|c|c|}
\hline Valor $\mathrm{p}^{*}$ & 0,674 & 0,575 & 0,826 & 0,652 & 0,631 \\
\hline \multicolumn{6}{|l|}{ Atividade física } \\
\hline \multirow[t]{2}{*}{$<150 \mathrm{mi} /$ semana } & $1,5(0,6-2,6)$ & $2,0(0,8-2,6)$ & $1,4(0,5-2,7)$ & $1,0(1,0-1,1)$ & $4,2(4,0-9,3)$ \\
\hline & $1,1(0,7-3,2)$ & $2,9(1,7-3,1)$ & $2,1(0,6-3,1)$ & $1,0(1,0-1,0)$ & $7,2(4,3-10,5)$ \\
\hline Valor $p^{* *}$ & 0,954 & 0,156 & 0,163 & 0,316 & 0,290 \\
\hline \multicolumn{6}{|l|}{ Tipo de diabetes } \\
\hline Tipo 1 ou gestacional & $1,1(1,0-2,0)$ & $1,8(1,4-3,1)$ & $0,8(0,2-2,4)$ & $1,0(1,0-1,0)$ & $4,6(3,7-8,3)$ \\
\hline Tipo 2 & $1,1(0,7-2,6)$ & $2,0(1,2-2,8)$ & $1,0(0,5-2,4)$ & $1,0(1,0-1,0)$ & $4,6(4,0-7,7)$ \\
\hline Valor $\mathrm{p}^{*}$ & 0,620 & 0,666 & 0,689 & 0,713 & 0,987 \\
\hline \multicolumn{6}{|l|}{ Tempo de diagnóstico } \\
\hline$<3$ anos & $1,1(0,7-1,5)$ & $2,2(1,4-3,15)$ & $1,2(0,2-2,5)$ & $1,0(1,0-1,0)$ & $5,2(3,9-8,2)$ \\
\hline$\geq 3$ anos & $1,8(0,7-2,7)$ & $1,5(0,9-2,7)$ & $0,8(0,7-2,1)$ & $1,0(1,0-1,0)$ & $4,7(3,9-8,4)$ \\
\hline Valor $p^{*}$ & 0,549 & 0,292 & 0,838 & 0,323 & 0,977 \\
\hline \multicolumn{6}{|c|}{ Acompanhamento nutricional } \\
\hline $\operatorname{Sim}$ & $2,0(0,9-2,7)$ & $2,9(1,6-3,5)$ & $1,8(1,1-2,6)$ & $1,0(1,0-1,0)$ & $8,5(4,5-9,7)$ \\
\hline Não & $1,1(0,7-2,4)$ & $2,0(1,1-2,7)$ & $0,8(0,4-2,0)$ & $1,0(1,0-1,0)$ & $4,4(3,7-7,4)$ \\
\hline Valor $\mathrm{p}^{*}$ & 0,447 & 0,049 & 0,060 & 0,652 & 0,072 \\
\hline$<50 \%$ das questões & $1,1(0,7-3,0)$ & $1,5(1,0-2,6)$ & $0,8(0,7-2,5)$ & $1,0(1,0-1,0)$ & $4,3(3,9-8,7)$ \\
\hline$\geq 50 \%$ das questões & $1,1(0,7-2,3)$ & $2,2(1,4-2,9)$ & $1,0(0,4-2,4)$ & $1,0(1,0-1,0)$ & $6,0(3,9-8,3)$ \\
\hline Valor $\mathrm{p}^{*}$ & 0,769 & 0,211 & 0,724 & 0,247 & 0,769 \\
\hline
\end{tabular}

Tabela 6 - Percentual de acertos sobre questões antes e após a intervenção.

\begin{tabular}{|c|c|c|c|}
\hline \multirow{2}{*}{ Questões } & \multicolumn{2}{|c|}{ \% de Acertos } & \multirow{2}{*}{ Valor $\mathrm{p}^{*}$} \\
\hline & Antes intervenção & Após intervenção & \\
\hline 1 & 65,0 & 75,0 & 0,727 \\
\hline 2 & 60,0 & 95,0 & 0,016 \\
\hline 3 & 50,0 & 90,0 & 0,021 \\
\hline 4 & 35,0 & 95,0 & $<0,001$ \\
\hline 5 & 40,0 & 90,0 & 0,006 \\
\hline 6 & 70,0 & 100,0 & - \\
\hline 7 & 65,0 & 100,0 & - \\
\hline 8 & 35,0 & 100,0 & - \\
\hline
\end{tabular}

da gordura na carótida. Desta forma, as dietas ricas em fibras são um componente importante no controle do diabetes. Com base nesses achados, aumentar a ingestão diária de fibras em $15 \mathrm{~g}$ para $35 \mathrm{~g}$ pode ser uma meta razoável que se espera reduzir o risco de mortalidade prematura em adultos com diabetes ${ }^{23}$.

$\mathrm{Na}$ UBS onde foi realizado o estudo não há presença de nutricionista. A orientação nutricional fornecida impactou positivamente, pois houve aumento do número de acerto nas questões, o que demonstra como a presença do nutricionista é essencial para auxiliar no tratamento do diabetes.

O consumo de uma dieta com poucas fontes de fibras alimentares pode estar associado a condições socioeconômicas, demográficas e nível de esco- laridade dos indivíduos, uma vez que indivíduos com renda mais elevada e maior nível de escolaridade tendem a consumir mais alimentos fontes desses nutrientes ${ }^{24}$. Em nosso estudo, não encontramos essa associação, mas foi verificado que os pacientes que realizavam acompanhamento com o nutricionista apresentaram um consumo maior de vegetais em relação aos outros pacientes. Notou-se que $65,8 \%$ dos avaliados não praticavam nenhum tipo de atividade física, sabe-se, porém, que o exercício físico é considerado importante fator para o controle do DM, principalmente, quando associado à alimentação adequada $^{25}$.

\section{CONCLUSÃO}

O nível de conhecimento a respeito de fibras e seus benefícios eram insuficientes nesse público o que influenciou no baixo consumo dos mesmos. Percebeu-se também, corre- 


\section{artigo}

Amorim, G.; Ferreira, D.C.; Miranda, V.O.; Santos, D.C.; Vieira, M.P.;

Conhecimento de indivíduos diabéticos sobre os benefícios e fontes da fibra alimentar

lação positiva entre acompanhamento nutricional e consumo de vegetais, o que evidencia a importância do acompanhamento e tratamento assertivo, justificando-se pelo fato de as demais variáveis estudadas não terem apresentado relação com o nível de conhecimento sobre fibras.

Esses fatores demonstram a importância da presença de um profissional nutricionista compondo as equipes multiprofissionais nas Unidades Básicas de Saúde, uma vez que esses poderão auxiliar os usuários a realizar adequações nos hábitos alimentares, assim como, conhecer e entender a função dos alimentos que poderão ser utilizados na prevenção e manejo da saúde.

\section{REFERÊNCIAS}

1.International Diabetes Federation. Atlas. 9 ed. About Diabetes: International Diabetes Federation, 2019.

2.Sociedade Brasileira de Diabetes. Diretriz da Sociedade Brasileira de Diabetes. São Paulo, 2019-2020.

3.Saraiva, J. F. K. et al. Diabetes mellitus no Brasil: características clínicas, padrão de tratamento e custos associados ao cuidado da doença. Jornal Brasileiro de Economia da Saúde: São Paulo, v. 8, n. 2, pág. 80-90, 2016.

4. Queres, J. F. M. et al. Avaliação do conhecimento e atitudes e sua relação com parâmetros de controle do Diabetes Mellitus. Journal of Health Connections, v. 2, n. 1, 2018.

5.Weickert M. O, Pfeiffer a. F. H. Impact of Dietary Fiber Consumption on Insulin Resistance and the Prevention of Type 2 Diabetes. J Nutr. 2018 1;148(1):7-12.

6.Davison KM, Temple NJ. Cereal fiber, fruit fiber, and type 2 diabetes: Explaining the paradox. J Diabetes Complications, v. 32, n. 2, p. 240-245.

7. Makki K, Deehan EC, Walter J, Bäckhed F. The Impact of Dietary Fiber on Gut Microbiota in Host Health and Disease. Cell Host Microbe. v. 13, n. ;23, 2018.

8.Macedo, T. M. B.; Schmourlo, G.; Viana, K. D. A. L. Fibra alimentar como mecanismo preventivo de doenças crônicas e distúrbios metabólicos. UNI: Imperatriz, n.2, p.67-77, 2012.

9.Silva, G. M. Elevada prevalência de inadequação do consumo de fibras alimentares em idosos e fatores associados: um estudo de base populacional. Revista brasileira de epidemiologia, v. 22, n. 19, 2019.

10.Magalhães, B.C. et al. Consumo de fibras alimentares entre indivíduos adultos em um supermercado de São Luis, Maranhão. Revista Pesquisa Saúde, São Luís, p. 137-140, 2016.

11.Bernaud, F.S.R; Rodrigues, T.C. Fibra alimentar - Ingestão adequada e efeitos sobre a saúde do metabolismo. Arquivos Brasileiros de endocrinologia e metabologia, Porto Alegre, p. 397405,2013.

12.Sociedade Brasileira de Diabetes. Diretriz da Sociedade Brasileira de Diabetes. São Paulo, 2019-2020.

13.Muniz, L.C. et al. Prevalência e fatores associados ao consumo de frutas, legumes e verduras entre adolescentes de escolas públicas de Caruaru, PE. Ciência \& Saúde Coletiva, Pelotas, p. 393-404, 2013.

14.Molz, P. et al. Relação do consumo alimentar de fibras e da carga glicêmica sobre marcadores glicêmicos, antropométricos e dietéticos em pacientes pré-diabéticos. Revista Epidemiologia
Controle Infectologia, Santa Cruz do Sul, v.5, n.3, p. 131-135, jul./ set. 2015.

15.Menezes, T. M.; Pedraza, D. F. Questionários de Frequência de Consumo Alimentar desenvolvidos e validados para população do Brasil: revisão da literatura. Ciência e saúde coletiva, v .20, n. 9, 2015.

16.Anunciação, P.C. et al. Avaliação do conhecimento sobre alimentação antes e após intervenção nutricional entre diabéticos tipo 2. 12. Revista Baiana de Saúde Pública. v.36, n.4, p.9861001, 2012.

17.Scagliusil, F.B. et al. Tradução, adaptação e avaliação psicométrica da Escala de Conhecimento Nutricional do National Health Interview Survey Cancer Epidemiology. Revista de Nutrição, 2006.

18.Mendonça, R.D. Efetividade de ações de promoção do consumo de frutas e hortaliças no Programa Academia da Saúde. 2016. 212p. Tese - Universidade Federal de Minas Gerais, MG, 2016

19.Campbell, K. J. et al. Home food availability mediates associations between mother nutrition knowledge and child diet. Appetite, London, v. 71. P. 1-6, 2013.

20.Guibu , I. A. Características principais dos usuários dos serviços de atenção primária à saúde no Brasil. Revista de Saúde Pública, v .51, suppl.2, 17s. Nov 13, 2017.

21.Fernandez, M. N; Cazelli, C; Teixeira, J.R. Gerenciamento do controle glicêmico do diabetes mellitus tipo dois na Estratégia de Saúde da Família. Hospital, Universitário Pedro Ernesto, Rio de janeiro-RJ, v. 15, n. 3, p. 218-226, julho/set2016.

22.Freitas, L.R.S et al. Evolução da prevalência do diabetes e deste associado à hipertensão arterial no Brasil: análise da Pesquisa Nacional por Amostra de Domicílios, 1998, 2003 e 2008. Epidemiologia e Serviços de Saúde, Brasília, v. 21, n. 1, 2012.

23. Reynolds A. N, Akerman A. P, Mann J. Dietary fibre and whole grains in diabetes management: Systematic review and meta-analyses. PLoS Med., v. 6, n. 17, .2020.

24.Cecílio, H. P. M. Comportamentos e comorbidades associados às complicações microvasculares do diabetes. Acta paulista de enfermagem, v.28, n.2, 2015.

25.Silva, F.M.; Steemburgo, T.; Azevedo, M.J.; Mello, V.D. Papel do índice glicêmico e da carga glicêmica na prevenção e no controle metabólico de pacientes com diabetes melito tipo 2. Arquivos Brasileiros de endocrinologia e metabologia, p.560-571, abril 2009. 